\section{Finding the Truth About Health Care Cost Drivers- Price Versus Utilization Factors}

The Blue Cross and Blue Shield Association sponsored a 400-page report released in October 2002 that concluded that 19\% of the increase in hospital costs between 1998 and 2001 was attributable to medical technology and 18\% to hospital consolidation. ${ }^{1}$ The Federation of American Hospitals, which represents 1,700 for-profit hospitals, responded by planning its own study of health care cost drivers, with a specific focus on insurance premiums. ${ }^{2}$ No doubt that both organizations will produce credible data. Observers will be left to interpret the data to find the truth.

A report released in July 2000 by the National Institute for Health Care Management (NIHCM) Research and Education Foundation found that the average patent life of new drug approvals in the middle 1980s until the present was effectively 13 to 15 years as a result of 6 laws enacted between 1983 and 1997 that strengthened the patents of brand drugs, extended their period of exclusivity, or eased the transfer of government discoveries to pharmaceutical companies. The report was critical of these federal protections that reduced generic drug competition, increased consumer prices, and helped make the drug industry the most profitable industry in the United States. NIHCM also found that $64 \%$ of "new" drug approvals were actually reformulations of existing drugs or combinations of existing drugs. ${ }^{3}$ The NIHCM study in 2000 was followed by a study released in May 2002 that found only 361 (35\%) of 1,035 new drugs approved by the U.S. Food and Drug Administration from 1989 through 2000 to be new molecular entities. ${ }^{4}$ The other 674 "new" drugs were existing drugs or modified versions of existing drugs. Only 153 drugs (15\%) were designated as "highly innovative drugs." ${ }^{\prime 5}$ Both studies were shots across the bow of the brandname pharmaceutical industry, represented by the National Pharmaceutical Council (NPC) and the Pharmaceutical Research and Manufacturers of America (PhRMA). NIHCM is funded largely by Blue Cross and Blue Shield health plans.

NIHCM has needled PhRMA and NPC in other ways. NIHCM reports in 1999 and 2002 attributed more of the large cost trend in prescription drug spending by health plans to "price" rather than to utilization. The July 9, 1999, report, "Factors Affecting the Growth of Prescription Drug Expenditures," prepared by Barents Group LLC for NIHCM, found that total spending on prescription drugs increased by an average annual $11 \%$ in the 5-year period from 1992 to 1997, twice the 5.5\% average annual increase for total health care spending. ${ }^{6}$ There was also acceleration in the increase in prescription drug spending, rising 8.7\% in $1993,9.0 \%$ in $1994,10.6 \%$ in $1995,13.2 \%$ in 1997 , and $18.4 \%$ in 1998 . This report pointed the finger at the drugs most heavily advertised to consumers as a major source of the increase in drug spending. The 1999 report from NIHCM was followed by the May 2001 report, "Prescription Drug Expenditures in 2000: The Upward Trend Continues," in which the $18.8 \%$ increase in outpatient prescription drug spending was attributed $42 \%$ to "an increase in the number of prescriptions dispensed," in other words, utilization, and 58\% to increase in "price," distributed $22 \%$ (about one third) to the "price of individual drugs" and 36\% to "the shift in the mix of drugs dispensed." The May 2002 report from NIHCM, "Prescription Drug Expenditures in 2001: Another Year of Escalating Costs," attributed the 17.1\% increase in drug spending in 2001 to a 39\% "increase in the number of prescriptions," $37 \%$ to "price increases," and $24 \%$ to a "shift to higher-cost drugs." So, NIHCM attributed 58\% of the $18.8 \%$ cost trend for prescription drugs in 2000 to price and $61 \%$ of the $17.1 \%$ cost trend in 2001 to price.

In this issue of the Journal, Gilberg, Laouri, Wade, and Isonaka examined drug utilization as determined from dispensing records (drug claims) in patients with medical claim diagnoses for asthma, congestive heart failure, depression, or common cold/upper respiratory tract infection. ${ }^{8}$ Along the way, the authors claim that "utilization" is the major driver for drug cost trends and, hence, the need for more examination of drug utilization patterns in the United States. Readers should note that this study was funded by the National Pharmaceutical Council, a champion of policy and interests of brand-name prescription drug manufacturers.

This battle between PhRMA/NPC and NIHCM regarding price versus utilization as the drivers of the prescription drug expenditure trend is no small matter, with barrages from both sides. Some of the back-and-forth can be reviewed at www.nihcm.org, including "The NIHCM Foundation Responds to PhRMA's Criticisms of the Report, 'Changing Patterns of Pharmaceutical Innovation."' One line of thinking appears to assume that prescription drug utilization is de facto medically necessary. If one makes this assumption, then the next argument is that any effort to reduce drug spending could have adverse health consequences, if utilization is, in fact, a sufficiently large percentage of the increase in drug spending. The third argument in this line of thinking is that price controls will not have much influence on total drug spending if price is a relatively small portion of the trend in drug spending. But NIHCM attributed 58\% of the 18.8\% cost trend for prescription drugs in 2000 to price and $61 \%$ of the $17.1 \%$ cost trend in 2001 to price. These findings have been supported by the work of others, ${ }^{10}$ including pharmacy benefit manager Express Scripts, which found 63\% of the 16.9\% cost trend in prescription drugs in 2001 to be attributable to price. Therefore, these 2 data sources showed utilization to account for $37 \%$ to $39 \%$ of the approximate $17 \%$ increase in prescription drug spending in 2001.

It is also important to take a temporal perspective of the price versus utilization debate in total prescription drug spending. In the trenches of drug benefit management in 2001-2003, price appears to be maintaining its prominent and dominant position, compared to utilization. Based on data in the IMS Health summary report released in February 2003, prescription drug sales in the United States in 2002 rose by $11.7 \%$, from $\$ 172.0$ billion in 
2001 to $\$ 192.2$ billion. ${ }^{11}$ Drug price inflation for existing drugs was $4 \%$ in 2002, twice the inflation rate in the general economy and more than one third of the total $11.7 \%$ increase in drug spending. Utilization as measured by the volume of prescriptions accounted for less than $40 \%$ of the increase in total sales. Drug "mix," defined as the use of newer, higher-cost drugs, accounted for the balance of the drug cost trend in 2002. In other words, inflation in prices of existing drugs, utilization, and the drug "mix" each accounted for about one third of the total increase in prescription drug spending in 2002.

Data from Verispan Scott-Levin for community pharmacy sales in calendar year 2002 show a more remarkable influence of price versus utilization in prescription drug sales. These data, based on prescriptions dispensed in 36,000 chain and independent pharmacies, mass-merchandisers, deep-discounters, and food stores, showed a 10\% increase in prescription drug sales in 2002 to $\$ 166.66$ billion, contributed $95 \%$ by price and about $5 \%$ by utilization. ${ }^{12}$ The average prescription price increased by $9.5 \%$ to $\$ 54.57$ in 2002, and volume increased $0.4 \%$ to 3.054 billion prescriptions.

Yet, the commonly accepted measure of utilization of prescription drugs, the prescription dispensed, has flaws, and the drug industry position, as presented by NPC and PhRMA, has merit in this regard. Unreported in the summary data for community pharmacy is the "size" of the average prescription as measured in days of therapy. Second, the relative growth in mailorder pharmacy sales in the United States over the last decade has contributed to growth in the size of the average prescription as measured in days of therapy. The customary mail-order prescription is nearly 3 times the size of the average prescription dispensed by community pharmacy, and a more precise measure of "utilization" would appear to be number of days of therapy rather than the number of prescriptions. At the least, this alternate measure of days of therapy would provide additional information about the trend of increased utilization of prescription drugs. ${ }^{13}$ Mail-order pharmacy accounted for about 120 million prescriptions and $\$ 8.5$ billion in sales in 1995 , or about $10 \%$ of the total pharmaceutical market. ${ }^{14}$ Mail-order pharmacy had an average annual growth rate of $20 \%$ over the 8-year period ended June 30 , 2001 , accounting for $16 \%$ of all prescription drug dollars in 2001. ${ }^{15}$ Data from IMS Health showed that mail-order pharmacy accounted for $17 \%$ of the prescription drug market in calendar year 2001 as measured by "retail" pharmacy sales (versus manufacturer sales). ${ }^{16}$ This growth in mail-order pharmacy as a percentage of total "retail" pharmacy sales would cause the prescription as a measure of utilization to understate the actual increase in utilization of prescription drugs and as a percentage of the total increase in prescription drug spending in the United States.

The proliferation of generic drug alternatives in the treatment of depression, heartburn, hypertension, and other important therapeutic categories increases the opportunities for managed care pharmacy to blunt the cost trend in prescription drugs. Overall, much of the opportunity appears to be unfulfilled. For example, the top 2 drugs by total spending in 2002 were atorvastatin at $\$ 6.1$ billion and simvastatin at $\$ 4.2$ billion, up 19\% and $16 \%$ from 2001, respectively. Both are brand drugs for treating dyslipidemia, a condition treated effectively in most patients by generic lovastatin at about $75 \%$ to $85 \%$ discount to the cost of the brand drug alternatives. Two of the other top 10 drugs in 2002 were sertraline at $\$ 2.5$ billion and paroxetine at $\$ 2.3$ billion, up $14 \%$ and $11 \%$ from 2001, respectively. These 2 drugs are therapeutic alternatives to generic fluoxetine, which has a managed care price about $90 \%$ less than sertraline or paroxetine. Stated in terms of population health, generic fluoxetine can treat about 10 patients with depression for the same managed care cost as treating 1 patient with either sertraline or paroxetine. Generic lovastatin can treat about 5 patients with dyslipidemia for the same managed care cost as 1 patient treated with simvastatin and about 4 patients with dyslipidemia for each patient treated with atorvastatin.

\section{Real-World Research in Diabetes Care and Protocols for Patient Privacy}

Two articles in this issue of the Journal derive from research in Veteran Affairs (VA) medical centers. Aside from the obvious predominance of males in these study populations, readers might also note the possibility of alternate explanations for the study findings in the effectiveness research in diabetes care by Duckworth, Marcelli, Padden, et al., using combination glyburide-metformin versus separate formulations of metformin and sulfonylurea. ${ }^{17}$ In this pre/post study of patients switched from separate sulfonylurea and metformin to combination glyburidemetformin, there was a significant increase in (a) the number of patients who received insulin (on separate sulfonylura and metformin), from $5(6.9 \%)$ in the preperiod to $19(26.4 \% ; P=0.0001)$ in the postperiod; (b) the number of patients who received additional oral antidiabetic agents, from $6(8.3 \%)$ in the preperiod to 15 (20.8\%; $P=0.04)$ in the postperiod; and (c) the mean dose of metformin, from $1,607 \mathrm{mg}$ in the preperiod to $1,750 \mathrm{mg}$ in the post period $(P=0.02)$. These factors create alternative explanations for the $0.6 \%$ improvement in hemoglobin $\mathrm{AlC}(P=0.002)$ at a mean follow-up of 196 days after the switch to combination glyburide-metformin tablets.

This study of combination glyburide-metformin suggests that there may be some synergy in the formulation of the combination product that is not explained by the simple addition of the 2 products, noting the possible alternate explanations for the findings. Also, there was a remarkable increase in the number of patients who experienced clinically significant hypoglycemia in the postswitch period, on the combination glyburide-metformin product. Eight patients experienced clinically significant hypoglycemia in the postperiod on the combination product versus 3 patients who experienced hypoglycemia in the preswitch period. This outcome may be related more to the significant increase in the number of patients who received concomitant insulin in the postswitch period rather than being attributable to the combination glyburide-metformin product. 